\title{
Predictive Value of Nephrometry Scores in Nephron-sparing Surgery: A Systematic Review and Meta-analysis
}

\author{
Alessandro Veccia ${ }^{a, b}$, Alessandro Antonelli $^{b}$, Robert G. Uzzo ${ }^{c}$, Giacomo Novara $^{d}$, \\ Alexander Kutikov ${ }^{c}$, Vincenzo Ficarra ${ }^{e}$, Claudio Simeone ${ }^{b}$, Vincenzo Mirone ${ }^{f}$, \\ Lance J. Hampton ${ }^{a}$, Ithaar Derweesh ${ }^{g}$, Francesco Porpiglia ${ }^{h}$, Riccardo Autorino ${ }^{a, *}$ \\ ${ }^{a}$ Division of Urology, VCU Health System, Richmond, VA, USA; ${ }^{\mathrm{b}}$ Urology Unit, ASST Spedali Civili Hospital, Brescia, Italy and Department of Medical and \\ Surgical Specialties, Radiological Science, and Public Health, University of Brescia, Italy; ${ }^{\mathrm{c}}$ Division of Urology, Fox Chase Cancer Center, Philadelphia, PA, USA; \\ ${ }^{\mathrm{d}}$ Department of Oncologic, Surgical and Gastrointestinal Sciences, Urologic Unit, University of Padua, Italy; ${ }^{\mathrm{e}}$ Department of Human and Pediatric Pathology \\ “Gaetano Barresi”, Urologic Section, University of Messina, Italy; ${ }^{\mathrm{f}}$ Department of Urology, Federico II University, Naples, Italy; ${ }^{\mathrm{g}}$ Department of Urology, UCSD \\ Health System, La Jolla, CA, USA; ${ }^{\mathrm{h}}$ Division of Urology, San Luigi Hospital, University of Turin, Orbassano, Italy
}

\section{Article info}

\section{Associate Editor: Malte Rieken}

Keywords:

Nephrometry score

Partial nephrectomy

Radical nephrectomy

Tumor complexity

\section{Abstract}

Context: Over the last decade, several nephrometry scores (NSs) have been introduced with the aim of facilitating preoperative decision making, planning, and counseling in the field of nephron-sparing surgery. However, their predictive role remains controversial.

Objective: To describe currently available nephrometry scores and to determine their predictive role for different outcomes by performing a systematic review and metaanalysis of the literature.

Evidence acquisition: PubMed, Embase $\mathbb{R}$, and Web of Science were screened to identify eligible studies. Identification and selection of the reports were conducted according to the Preferred Reporting Items for Systematic Reviews and Meta-analyses (PRISMA). A pooled analysis of NS predictive role of intraoperative, postoperative, oncological, and functional outcomes was performed. Odds ratio was considered the effect size. All the analyses were performed using Stata 15.0, and statistical significance was set at $p \leq 0.05$.

Evidence synthesis: Overall, 51 studies meeting our inclusion criteria were identified and considered for the analysis. Except for one prospective randomized trial, all the studies were retrospective. All the studies were found to be of intermediate quality, except for one of high quality. Most studies assessed the predictive role of the RadiusExophytic/Endophytic-Nearness-Anterior/Posterior-Location (RENAL) and Preoperative Aspects and Dimensions Used for an Anatomical (PADUA) scores, mostly regarding complications after nephron-sparing surgery. RENAL was an independent predictor of an on-clamp procedure $(p<0.001)$. Mayo Adhesive Probability score was related to adhesive perinephric fat $(p=0.005)$. Continuous and high-complexity RENAL scores were predictors of warm ischemia time (WIT; $p=0.006$ and $p<0.001$, respectively). Continuous $(p<0.001)$ and high-complexity $(p<0.001)$ PADUA scores were related to WIT. Continuous and high-complexity RENAL scores were predictors of overall complications ( $p=0.002$ and $p<0.001$, respectively). PADUA score was related to complications both as continuous $(p<0.001)$ and as a categorical value $(p<0.002)$. The RENAL scores $R=3(p=0.008), E=2(p=0.039)$, and hilar location $(p=0.006)$ were predictors of histological malignancy. Continuous and categorical RENAL scores were independent

\footnotetext{
* Corresponding author. Division of Urology, VCU Health, PO Box 980118, Richmond, VA 23298-0118, USA. Tel. +1-804-828-5320, Fax: +1-804-828-2157. E-mail address: ricautor@gmail.com (R. Autorino).
}

https://doi.org/10.1016/j.euf.2019.11.004

2405-4569/@ 2019 European Association of Urology. Published by Elsevier B.V. All rights reserved. 
predictors of an estimated glomerular filtration rate (eGFR) increase ( $p=0.006$ and $p<0.001$, respectively). The Diameter-Axial-Polar score $(p=0.018)$ and Peritumoral Artery Scoring System (PASS; $p=0.02$ ) were also independent predictors.

Conclusions: The literature regarding nephrometry scoring systems is sparse, and mostly focused on RENAL and PADUA, which are easy to calculate and have a good correlation with most outcomes. Renal Pelvic Score is the best predictor of pelvicalyceal entry/repair and urine leak, whereas Surgical Approach Renal Ranking and PASS strongly predict surgical approach and renal function variation, respectively. Other nephrometry scores based on mathematical models are limited by their complexity, and they lack evidence supporting their predictive value.

Patient summary: We reviewed the medical literature regarding the use and value of socalled "nephrometry scores," which are scoring systems based on radiological imaging and made to grade the complexity of a renal tumor. We analyzed whether these scoring systems can predict some of the outcomes of patients undergoing surgical removal of renal tumors.

(c) 2019 European Association of Urology. Published by Elsevier B.V. All rights reserved.

\section{Introduction}

With the establishment of nephron-sparing surgery (NSS) as the preferred treatment option for the management of renal masses [1], the past decade has witnessed the development of "nephrometry scores." In 2009, the Radius-Exophytic/Endophytic-Nearness-Anterior/Posterior-Location (RENAL) [2] and the Preoperative Aspects and Dimensions Used for an Anatomical (PADUA) [3] systems were introduced with the common aim of objectifying the anatomical complexity of a renal mass, assisting in surgical decision making, and facilitating outcome assessment [4]. Since then, several other scoring systems have been conceptualized and reported in an effort to improve the predictive value and promote clinical applicability (Fig. 1) [2,3,520]. While comparisons among the different systems remain sparse, RENAL and PADUA remain the most known and used ones in the literature [21,22].

Notwithstanding this significant research effort, it remains unclear what is the uptake of these scoring systems in daily clinical practice and their predictive value. The aim of the present study is to assess, in a systematic fashion, the entire spectrum of currently available nephrometry scores and their performance in the prediction of clinical outcomes in patients undergoing NSS.

\section{Evidence acquisition}

\section{1. $\quad$ Literature search}

After establishing a study protocol, two authors (A.V. and A.A.) performed an independent literature research on PubMed, Embase $\AA$, and Web of Science to identify relevant studies up to April 2019 (Supplementary material). It was filtered to include original articles only, while conference abstract, conference paper, reviews, letters, notes, editorials, and book chapters were excluded. Identification and selection of the studies was conducted according to the Preferred Reporting Items for Systematic Reviews and Meta-analyses (PRISMA) statement (www. prisma-statement.org; Supplementary Fig. 1) [23,24]. Title and abstracts were first reviewed to ascertain whether they would potentially follow the inclusion criteria (studies on nephrometry score reporting multivariate logistic regression analyses expressed by odds ratio [OR]). A fulltext analysis was performed to confirm inclusion. References of collected reports were manually reviewed to find additional studies of interest. The study protocol was registered on PROSPERO (CRD42019133331).

\subsection{Assessment of study quality}

We classified each study according to the level of evidence [25]. The quality of the studies was determined using the Newcastle-Ottawa Scale for nonrandomized controlled trials [26]. A total score of $\leq 5$ was considered low quality, 6-7 intermediate quality, and 8-9 high quality. Jadad scale was deemed suitable for evaluating the quality of the randomized studies [27].

\subsection{Data extraction and analysis}

OR and confidence interval ( $\mathrm{CI}$ ) were collected to assess the predictive value of nephrometry scores to predict surgical strategy (minimally invasive surgery [MIS] vs open, partial nephrectomy [PN] vs radical nephrectomy [RN], or onclamp resection), prolonged warm ischemia time (WIT; defined as $>20 \mathrm{~min}$ ), adhesive perinephric fat, overall and major complications, conversion to RN, pelvicalyceal system entry/repair, urine leak, malignancy, high-grade tumor, new-onset chronic kidney disease (CKD), renal function variation, and trifecta achievement.

Nephrometry scores were considered continuous and/or categorical (high complexity). OR and $\mathrm{CI}$ lower and upper limit logarithms were calculated. The results allowed us to obtain the standard error. LogOR and $\log \mathrm{CI}$ were pooled to obtain the effect size of the variables. Heterogeneity among the studies was weighted according to random effect [28]. If pooled analysis included $<25$ studies, a small sample size bias was established according to Egger's regression test [29]. A nonstatistically significant $p$ value was indicative of the absence of a small sample size bias. All the analyses have been performed using Stata 15.0 (StataCorp 2017, Stata Statistical Software: release 15; StataCorp LLC, College Station, TX, USA), and statistical significance was set at 

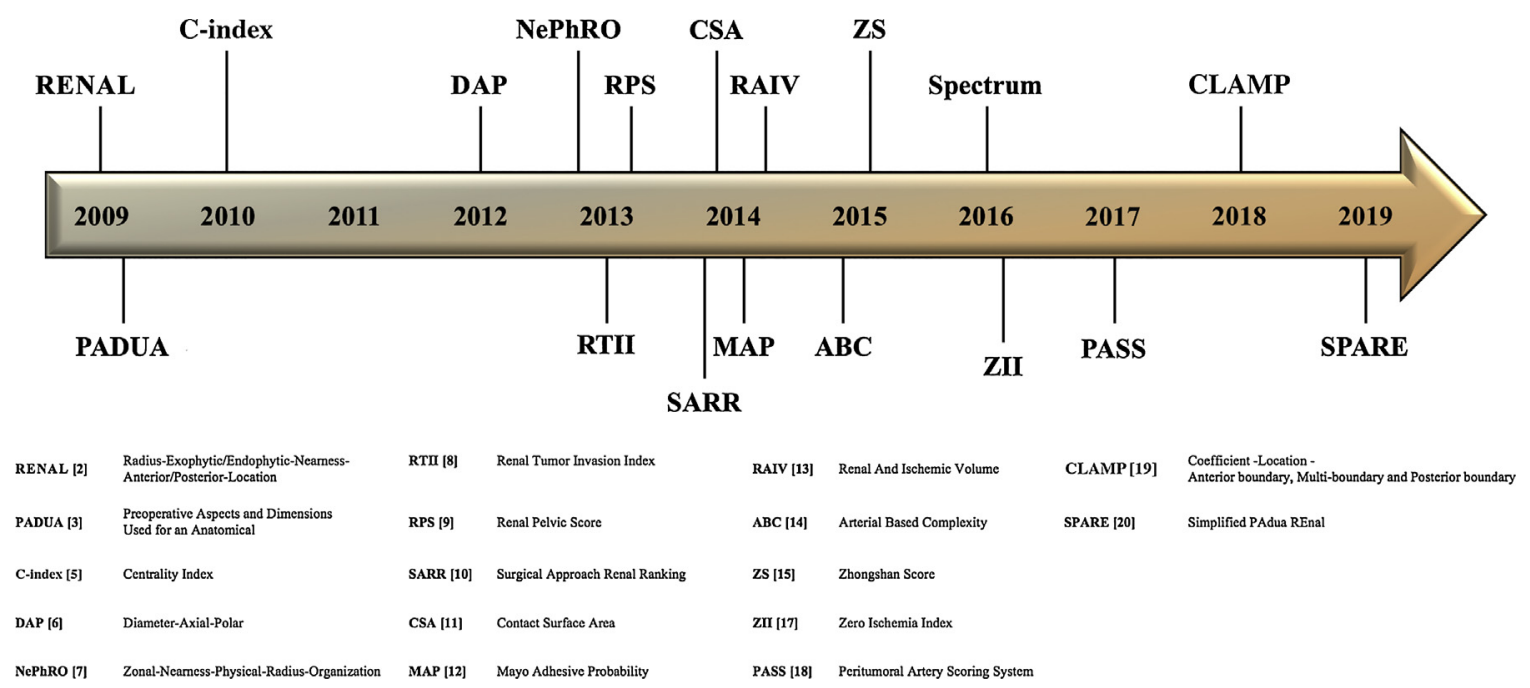

Fig. 1 - Nephrometry scores.
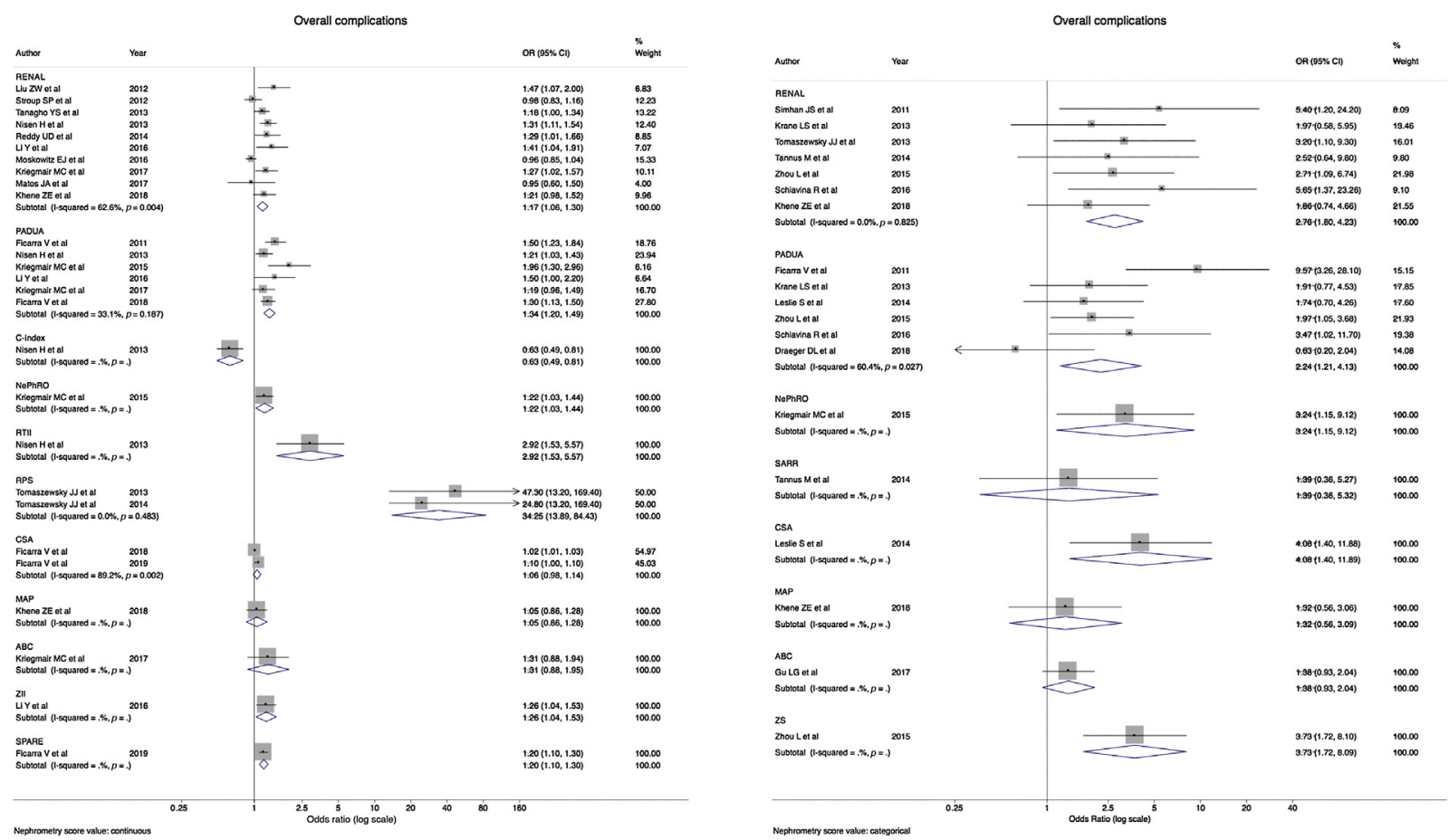

Fig. 2 - Predictive value of nephrometry score for overall complications. $\mathrm{ABC}=$ Arterial Based Complexity; $\mathrm{CI}=$ confidence interval; $\mathrm{C}$-index $=\mathrm{Central}$ Index; CSA = Contact Surface Area; MAP = Mayo Adhesive Probability; NePhRO = Nearness-Physical-Radius-Organization; OR = odds ratio; PADUA = Preoperative Aspects and Dimensions Used for an Anatomical; RENAL = Radius-Exophytic/Endophytic-Nearness-Anterior/Posterior-Location; RPS = Renal Pelvic Score; RTII = Renal Tumor Invasion Index; SARR = Surgical Approach Renal Ranking; SPARE = Simplified PAdua REnal-; ZII = Zero Ischemia Index; ZS = Zhongshan Score.

$p \leq 0.05$. A detailed description of statistical codes is reported in the Supplementary material.

\section{Evidence synthesis}

\subsection{Nephrometry scores}

Currently available nephrometry scores can arbitrarily be grouped into those based on a visual anatomical assessment of a renal mass and those based on a mathematical assessment.

3.1.1. Visual anatomical assessment-based scores

Most of the scores fall into this group as they are based on an immediate visual assessment. The RENAL [2] and PADUA [3] scores evaluate the tumor location, its degree of penetration within the kidney, and its relationship with the pelvicalyceal system. The Diameter-Axial-Polar(DAP) score establishes the 
renal mass dimension and distance from two referring lines: axial and polar lines [6]. The Zonal Nearness-Physical-RadiusOrganization (NePhRO) score presents five parameters that mirror those of the RENAL and PADUA scores. Differently, it divides the kidney into three zones (zone 1: kidney parenchyma; zone 2 : medullary and sinus; and zone 3 : collecting system and hilum) and adopts another dimensional scale to establish renal mass dimension [7]. Differently, the Renal Pelvic Score (RPS) departs from the abovementioned scores. Indeed, it assesses the presence of an intra- or extrarenal pelvis referring to a sagittal line that crosses the kidney hilum [9]. Another score, the Surgical Approach Renal Ranking (SARR), presents the same features as those of the RENAL, PADUA, and Zonal NePhRO scores, but it provides a scoring system from 0 to 4 , which allows obtaining more granular stratification of renal masses [10]. Most of the scores consider the longitudinal position of the tumor, while the Zhongshan score takes into account the transversal tumor position: lateral, central, and medial [15]. Recently, the Simplified PAdua REnal (SPARE) nephrometry system merged the main features of both the nephrometry scores to create a tumor rim location, renal sinus involvement, exophytic rate, and maximum tumor size-based score [20]. The Arterial Based Complexity $(A B C)$ scoring system considers the vascular involvement by the tumor. The four categories assessed $(1,2,3 S$, and $3 \mathrm{H}$ ) are linked to the neoplasm contact with interlobular or arcuate, interlobar, segmental, or hilum arteries, respectively [14]. Another vasculature-based score is the Peritumoral Artery Scoring System (PASS) [18]. It is a three-dimensionbased score that ranks tumor dissection difficulty according to the number and diameter of peritumoral arteries. Differently from the aforementioned scores, the Mayo Adhesive Probability (MAP) score evaluates the perinephric fat thickness as a mean to predict its adhesion to the kidney, which could translate into a more challenging resection [12].

\subsubsection{Mathematical assessment-based scores}

This category is based either on a visual or a mathematical assessment of the tumor, requiring detailed imaging analysis. The first one, the Centrality Index (C-index) classifies the tumor complexity according to its mathematical distance from the center of the kidney: $\sqrt{x^{2}+y^{2}}=c ; d / 2=r$; $c / r C$-index [5]. Less complex is the Renal Tumor Invasion Index (RTII), which is the ratio of the maximal invasion of the tumor from the surface of the kidney into the parenchyma and the parenchymal thickness of the kidney just beside the tumor $(I / P)[8]$. Other two scores calculate matematically the tumor Contact Surface Area (CSA) and the Renal And Ischemia Volume (RAIV), with both using the mass radius and diameter measurements. In addition, the RAIV requires the measurement of the resected and ischemized renal parenchyma cross section [11-13]. Similarly, the Zero Ischemia Index (ZII) represents the result of the product of the tumor diameter and its depth within the kidney parenchyma [17]. The only score assessing the vascular complexity is the Coefficient, Location, Anterior boundary, Multi-boundary, and Posterior boundary (CLAMP) score. This three-dimensional (3D) imaging-based score evaluates the anatomy of the arteries that feed the renal mass. This tool could predict the effectiveness of segmental artery clamping through the following mathematical formula:
$(X+Y)_{1} \times 1+(X+Y)_{2} \times \frac{1}{2}+\ldots(X+Y)_{x} \times \frac{1}{2}$, where $(X+Y)_{x}=$ ranking number of the target artery feeding the tumor [19]. Finally, the Spectrum score is a pure mathematic score that allows evaluation of the acute ipsilateral renal dysfunction based on renal scintigraphy measurements and serum creatinine levels [16].

\subsection{Description of included studies, and quality and bias assessment}

Overall, 51 studies meeting our inclusion criteria were identified and considered for the analysis [8-11,15,17,18,20,30-72]. Except for one prospective randomized trial [71], all the studies were retrospective. All the studies were found to be of intermediate quality, except for one of high quality [59] (Table 1). The majority of the studies assessed the predictive role of the RENAL and PADUA scores, mostly regarding complications after NSS (Supplementary Fig. 2 and 3).

A small sample bias was found in studies assessing WIT, overall and major complications, and pelvicalyceal system entry/repair. No obvious biases were marked regarding the other outcomes assessed (Supplementary Table 2).

\subsection{Prediction of outcomes}

\subsubsection{Surgical strategy}

Prediction of the use of an MIS approach was evaluated in only two studies [36,52]. Their pooled analysis failed to show RENAL as a predictor (Table 2 and the Supplementary material). The MAP score was assessed in only one study [52], and it also failed to demonstrate a predictive role.

Regarding the decision to perform PN versus RN, this was assessed in two studies, one for RENAL and SARR [10], and the other for PADUA and RTII [62]. No pooled analysis could be done. Only RENAL (OR 30.45; 95\% CI: 8.73, 106.1; $p<$ 0.001 ) and SARR (OR 39.53; 95\% CI: 10.55, 148; $p<0.001$ ) showed predictive values (Table 2 and the Supplementary material).

A cumulative analysis of three available studies [59,71,72] showed RENAL as an independent predictor of on-clamp resection (OR: 1.55; 95\% CI: 1.23, 1.95; $p<0.001$ ). Each of the PADUA, ABC [59], and MAP [72] scores was assessed in only one study and associated with on-clamp technique (all $p<0.001$; Table 2 and the Supplementary material).

\subsubsection{Adhesive perinephric fat}

Cumulative analysis of the two studies [46,58] reporting on this outcome demonstrated the MAP score to be an independent predictor of adhesive perinephric fat (OR: 1.98; 95\% CI: $1.23,3.18 ; p=0.005$; Table 2 and the Supplementary material).

\subsubsection{Warm ischemia time}

A pooled analysis of five studies demonstrated that the RENAL score as continuous (OR: 1.53; 95\% CI: 1.13, 2.06; $p=0.006$ ) [35-38,59] or categorical (high complexity; OR: 9.29; 95\% CI: 5.37, 16.06; $p<0.001)$ [35,53] is an 
Table 1 - Characteristics of the studies included.

\begin{tabular}{|c|c|c|c|c|c|c|c|c|c|c|c|}
\hline Author & $\begin{array}{l}\text { Nephrometry } \\
\text { score }\end{array}$ & Year & Journal & Institution & Study design & Patients & $\begin{array}{l}\text { Surgical } \\
\text { procedure }\end{array}$ & $\begin{array}{l}\text { Surgical } \\
\text { technique }\end{array}$ & $\begin{array}{l}\text { Nephrometry score } \\
\text { correlations assessed }\end{array}$ & LE & SQ \\
\hline \multirow[t]{4}{*}{ Mottrie et al [30] } & PADUA & 2011 & World J Urol & Single & Retrospective analysis & 62 & PN & Robotic & Warm ischemia time $\geq 20 \mathrm{~min}$ & 3 & $* * * * * * *$ \\
\hline & & & & & & & & & Perioperative complications & & \\
\hline & & & & & & & & & Overall complications & & \\
\hline & & & & & & & & & Pelvicalyceal repair & & \\
\hline \multirow[t]{2}{*}{ Kutikov et al [31] } & RENAL & 2011 & Eur Urol & Single & Retrospective analysis & 525 & PN & NA & Malignant histology & 3 & $* * * * * * *$ \\
\hline & & & & & & & & & High-grade histology & & \\
\hline \multirow[t]{3}{*}{ Simhan et al [32] } & RENAL & 2011 & Eur Urol & Single & Retrospective analysis & 390 & PN & Open & Major complications & 3 & $* * * * * * *$ \\
\hline & & & & & & & & Laparoscopic & & & \\
\hline & & & & & & & & Robotic & & & \\
\hline \multirow{2}{*}{ Ficarra et al [33] } & PADUA & 2011 & Eur Urol & Multiple & Retrospective analysis & 349 & PN & Robotic & Warm ischemia time $\geq 20 \mathrm{~min}$ & 3 & $* * * * * * *$ \\
\hline & & & & & & & & & Overall complications & & \\
\hline \multirow[t]{2}{*}{ Liu et al [34] } & RENAL & 2012 & World J Urol & Single & Retrospective analysis & 179 & PN & Laparoscopic & Overall complications & 3 & $* * * * * * *$ \\
\hline & & & & & & & & Robotic & & & \\
\hline \multirow[t]{2}{*}{ Mayer et al [35] } & RENAL & 2012 & Urology & Single & Retrospective analysis & 67 & PN & Laparoscopic & Warm ischemia time & 3 & $* * * * * * *$ \\
\hline & & & & & & & & Robotic & Collecting system entry & & \\
\hline \multirow[t]{4}{*}{ Stroup et al [36] } & RENAL & 2012 & Urology & Multiple & Retrospective analysis & 284 & PN & Open & Undergoing MIS vs OPN & 3 & $* * * * * * *$ \\
\hline & & & & & & & & Laparoscopic & Overall complications & & \\
\hline & & & & & & & & Robotic & Major complications & & \\
\hline & & & & & & & & & Urine leak & & \\
\hline \multirow{2}{*}{ Long et al [37] } & RENAL & 2012 & BJU Int & Single & Retrospective analysis & 177 & PN & Open & Conversion to RN & 3 & $* * * * * * *$ \\
\hline & & & & & & & & Laparoscopic & & & \\
\hline \multirow[t]{2}{*}{ Kopp et al [38] } & RENAL & 2012 & Urology & Single & Retrospective analysis & 228 & PN & Open & Warm ischemia time $\geq 20 \mathrm{~min}$ & 3 & $* * * * * * *$ \\
\hline & & & & & & & & & De novo CKD development & & \\
\hline \multirow[t]{2}{*}{ Mullins et al [39] } & RENAL & 2012 & $J$ Urol & Single & Retrospective analysis & 671 & PN & Robotic & Malignant histology & 3 & $* * * * * * *$ \\
\hline & & & & & & & & & High-grade histology & & \\
\hline Tanagho et al [40] & RENAL & 2013 & Urology & Multiple & Retrospective analysis & 886 & PN & Robotic & Perioperative complications & 3 & $* * * * * * *$ \\
\hline \multirow[t]{3}{*}{ Mehrazin et al [41] } & RENAL & 2013 & BJU Int & Multiple & Retrospective analysis & 322 & PN & Open & $\begin{array}{l}\text { eGFR }<60 \mathrm{ml} / \mathrm{min} / 1.73 \mathrm{~m}^{2} \text { in } \\
\text { the last follow-up }\end{array}$ & 3 & $* * * * * *$ \\
\hline & & & & & & & & Laparoscopic & & & \\
\hline & & & & & & & & Robotic & & & \\
\hline \multirow[t]{4}{*}{ Nisen et al [8] } & RENAL & 2013 & Scand J Urol & Single & Retrospective analysis & 285 & PN & Open & Any-grade complications & 3 & $* * * * * * *$ \\
\hline & PADUA & & & & & & & Laparoscopic & & & \\
\hline & C-index & & & & & & & Robotic & & & \\
\hline & RTII & & & & & & & & & & \\
\hline \multirow[t]{2}{*}{ Krane et al [42] } & RENAL & 2013 & BJU Int & Single & Retrospective analysis & 233 & PN & Robotic & Any complication & 3 & $* * * * * * *$ \\
\hline & PADUA & & & & & & & & Major complication & & \\
\hline \multirow[t]{2}{*}{ Tomaszewski et al [9] } & RENAL & 2013 & Eur Urol & Single & Retrospective analysis & 255 & PN & Open & Urine leak & 3 & $* * * * * * *$ \\
\hline & RPS & & & & & & & Robotic & & & \\
\hline \multirow[t]{3}{*}{ Tannus et al [10] } & RENAL & 2014 & $J$ Endourol & Single & Prospective analysis & 80 & PN & Open & Surgical approach selection & 2 & $* * * * * * *$ \\
\hline & SARR & & & & & & $\mathrm{RN}$ & Laparoscopic & General complications & & \\
\hline & & & & & & & & Robotic & & & \\
\hline \multirow[t]{3}{*}{ Antonelli et al [43] } & RENAL & 2014 & Clin Genitourin Cancer & Multiple & Retrospective analysis & 506 & PN & Open & Malignancy & 3 & $* * * * * * *$ \\
\hline & & & & & & & & Laparoscopic & High-grade features & & \\
\hline & & & & & & & & Robotic & & & \\
\hline \multirow[t]{5}{*}{ Leslie et al [11] } & PADUA & 2014 & Eur Urol & Single & Retrospective analysis & 200 & PN & Laparoscopic & Operative time $\geq 4 \mathrm{~h}$ & 3 & $* * * * * * * *$ \\
\hline & CSA & & & & & & & Robotic & Estimated blood loss $>500 \mathrm{ml}$ & & \\
\hline & & & & & & & & & Overall complications & & \\
\hline & & & & & & & & & Length of stay $\geq 4 \mathrm{~d}$ & & \\
\hline & & & & & & & & & Warm ischemia time & & \\
\hline
\end{tabular}




\begin{tabular}{|c|c|c|c|c|c|c|c|c|c|c|c|}
\hline Author & $\begin{array}{l}\text { Nephrometry } \\
\text { score }\end{array}$ & Year & Journal & Institution & Study design & Patients & $\begin{array}{l}\text { Surgical } \\
\text { procedure }\end{array}$ & $\begin{array}{l}\text { Surgical } \\
\text { technique }\end{array}$ & $\begin{array}{l}\text { Nephrometry score } \\
\text { correlations assessed }\end{array}$ & LE & SQ \\
\hline \multirow[t]{2}{*}{ Tomaszewski et al [44] } & RENAL & 2014 & Urology & Single & Retrospective analysis & 831 & $\mathrm{PN}$ & Open & Urine leak & 3 & ********** \\
\hline & RPS & & & & & & & Robotic & & & \\
\hline \multirow{2}{*}{ Reddy et al [45] } & RENAL & 2014 & Ann $R$ Coll Surg Engl & Single & Retrospective analysis & 128 & PN & Open & Postoperative complications & 3 & *********1 \\
\hline & & & & & & & & Laparoscopic & & & \\
\hline \multirow[t]{2}{*}{ Kocher et al [46] } & RENAL & 2014 & BJU Int & Single & Retrospective analysis & 245 & PN & Laparoscopic & Adherent perinephric fat & 3 & ********* \\
\hline & MAP & & & & & & & Robotic & & & \\
\hline \multirow[t]{2}{*}{ Ball et al [47] } & RENAL & 2014 & Urol Oncol & Multiple & Retrospective analysis & 1009 & PN & NA & Malignancy & 3 & ********* \\
\hline & & & & & & & & & Unfavorable pathology & & \\
\hline \multirow[t]{3}{*}{ Zhou et al [15] } & RENAL & 2015 & Medicine (Baltimore) & Single & Prospective study & 1231 & PN & NA & Overall complications & 2 & ******** \\
\hline & PADUA & & & & & & $\mathrm{RN}$ & & & & \\
\hline & $\mathrm{ZS}$ & & & & & & & & & & \\
\hline \multirow[t]{3}{*}{ Kwon et al [48] } & RENAL & 2015 & Ann Surg Oncol & Single & Retrospective analysis & 266 & PN & NA & GFR reduction & 3 & ******* \\
\hline & PADUA & & & & & & & & New-onset CKD & & \\
\hline & C-index & & & & & & & & & & \\
\hline \multirow[t]{3}{*}{ Li et al [49] } & DAP & 2015 & Medicine (Baltimore) & Single & Retrospective analysis & 237 & PN & Open & Warm ischemia time $\geq 20 \mathrm{~min}$ & 3 & $* * * * * *$ \\
\hline & & & & & & & & Laparoscopic & eGFR decline $>10 \%$ & & \\
\hline & & & & & & & & Robotic & & & \\
\hline Kriegmair et al [50] & NePhRO & 2015 & World J Urol & Single & Retrospective analysis & 200 & PN & Open & Perioperative complications & 3 & $* * * * * *$ \\
\hline Kriegmair et al [51] & PADUA & 2015 & Biomed Res Int & Single & Retrospective analysis & 233 & PN & Open & Major complications & 3 & 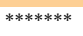 \\
\hline \multirow[t]{2}{*}{ Sharma et al [52] } & RENAL & 2016 & Indian J Urol & Single & Retrospective analysis & 119 & PN & Open & Predictors OPN & 3 & $* * * * * *$ \\
\hline & MAP & & & & & & & Robotic & & & \\
\hline \multirow[t]{3}{*}{ Raheem et al [53] } & PADUA & 2016 & BJU Int & Single & Retrospective analysis & 295 & PN & Open & Trifecta achievement & 3 & ******** \\
\hline & & & & & & & & Laparoscopic & & & \\
\hline & & & & & & & & Robotic & & & \\
\hline \multirow[t]{4}{*}{ Schiavina et al [54] } & RENAL & 2016 & BJU Int & Multiple & Retrospective analysis & 277 & PN & Robotic & Warm ischemia time $\geq 20 \mathrm{~min}$ & 3 & ******** \\
\hline & PADUA & & & & & & & & Need for UCS repair & & \\
\hline & & & & & & & & & Overall complications & & \\
\hline & & & & & & & & & Major complications & & \\
\hline Ricciardulli et al [55] & PADUA & 2016 & Urologia & Single & Retrospective analysis & 402 & PN & Laparoscopic & Warm ischemia time $\geq 20 \mathrm{~min}$ & 3 & ******** \\
\hline \multirow[t]{4}{*}{ Li et al [17] } & RENAL & 2016 & World J Urol & Single & Retrospective analysis & 149 & PN & Open & Estimated blood loss $\geq 500 \mathrm{ml}$ & 3 & $* * * * * * *$ \\
\hline & PADUA & & & & & & & Laparoscopic & Operative time $>2 \mathrm{~h}$ & & \\
\hline & ZII & & & & & & & Robotic & Overall complications & & \\
\hline & & & & & & & & & eGFR decrease > $10 \%$ & & \\
\hline \multirow[t]{2}{*}{ Moskowitz et al [56] } & RENAL & 2016 & $J$ Endourol & Multiple & Retrospective analysis & 1139 & PN & Robotic & Overall complications & 3 & $* * * * * * *$ \\
\hline & & & & & & & & & Major complications & & \\
\hline \multirow[t]{2}{*}{ Kara et al [57] } & RENAL & 2017 & J Urol & Single & Retrospective analysis & 1023 & PN & Robotic & Conversion to RN & 3 & $* * * * * * *$ \\
\hline & & & & & & & $\mathrm{RN}$ & & & & \\
\hline Martin et al [58] & MAP & 2017 & Urology & Single & Retrospective analysis & 86 & PN & NA & Adherent perinephric fat & 3 & ******** \\
\hline \multirow[t]{4}{*}{ Kriegmair et al [59] } & RENAL & 2017 & J Surg Oncol & Single & Retrospective analysis & 300 & PN & Open & Complications & 3 & $* * * * * * * * *$ \\
\hline & PADUA & & & & & & & Robotic & On-clamp excision & & \\
\hline & $\mathrm{ABC}$ & & & & & & & & Ischemia time & & \\
\hline & & & & & & & & & Opening of the CS & & \\
\hline \multirow[t]{2}{*}{ Matos et al [60] } & RENAL & 2017 & Int Braz J Urol & Single & Retrospective analysis & 71 & PN & Open & Major complications & 3 & $* * * * * * *$ \\
\hline & & & & & & & $\mathrm{RN}$ & Laparoscopic & & & \\
\hline \multirow[t]{2}{*}{ Gu et al [61] } & $A B C$ & 2017 & J Surg Oncol & Single & Retrospective analysis & 350 & PN & Laparoscopic & Overall complications & 3 & ********* \\
\hline & & & & & & & & Robotic & Minor complications & & \\
\hline \multirow[t]{4}{*}{ Zhang et al [18] } & RENAL & 2017 & Sci Rep & Single & Retrospective analysis & 220 & PN & NA & GFR percent decline $\geq 10 \%$ & 3 & ******** \\
\hline & PADUA & & & & & & & & GFR percent decline $\geq 20 \%$ & & \\
\hline & RAIV & & & & & & & & & & \\
\hline & $\mathrm{ABC}$ & & & & & & & & & & \\
\hline
\end{tabular}




\begin{tabular}{|c|c|c|c|c|c|c|c|c|c|c|c|}
\hline Author & $\begin{array}{l}\text { Nephrometry } \\
\text { score }\end{array}$ & Year & Journal & Institution & Study design & Patients & $\begin{array}{l}\text { Surgical } \\
\text { procedure }\end{array}$ & $\begin{array}{l}\text { Surgical } \\
\text { technique }\end{array}$ & $\begin{array}{l}\text { Nephrometry score } \\
\text { correlations assessed }\end{array}$ & LE & SQ \\
\hline & PASS & & & & & & & & & & \\
\hline \multirow{2}{*}{ Tornberg et al [62] } & PADUA & 2017 & Scand J Surg & Single & Retrospective analysis & 915 & PN & NA & Performing PN & 3 & $* * * * * * *$ \\
\hline & RTII & & & & & & RN & & & & \\
\hline Takagi et al [63] & RENAL & 2017 & $J$ Endourol & Single & Matched-pair analysis & 227 & PN & Robotic & eGFR decrease $10 \%$ & 3 & $* * * * * *$ \\
\hline \multirow{2}{*}{ Correa et al [64] } & RENAL & 2018 & Clin Genitourin Cancer & Single & Retrospective analysis & 334 & PN & NA & Malignancy & 3 & ${ }^{* * * * * * * *}$ \\
\hline & & & & & & & & & High-grade features & & \\
\hline \multirow[t]{3}{*}{ Draeger et al [65] } & PADUA & 2018 & Turk J Urol & Single & Retrospective analysis & 213 & PN & NA & Symptoms & 3 & ${ }^{* * * * * * * *}$ \\
\hline & & & & & & & & & CT stage $\mathrm{T} 1 \mathrm{~b}, \mathrm{~T} 2$ & & \\
\hline & & & & & & & & & Complications & & \\
\hline \multirow[t]{3}{*}{ Petros et al [66] } & RENAL & 2018 & Urology & Single & Retrospective analysis & 90 & PN & Open & Conversion to RN & 3 & $* * * * * * *$ \\
\hline & & & & & & & RN & Laparoscopic & & & \\
\hline & & & & & & & & Robotic & & & \\
\hline \multirow{4}{*}{ Khene et al [67] } & RENAL & 2018 & Urol Oncol & Single & Retrospective analysis & 500 & PN & NA & Conversion to $\mathrm{RN}$ & 3 & ${ }^{* * * * * * * *}$ \\
\hline & MAP & & & & & & & & Overall complications & & \\
\hline & & & & & & & & & Major complications & & \\
\hline & & & & & & & & & Trifecta achievement & & \\
\hline Wang et al [68] & RENAL & 2018 & Urol Oncol & Single & Retrospective analysis & 337 & PN & NA & eGFR stabilization or variation & 3 & $* * * * * *$ \\
\hline \multirow[t]{3}{*}{ Ficarra et al [69] } & PADUA & 2018 & BJU Int & Multiple & Retrospective analysis & 531 & PN & Open & Postoperative complications & 3 & $* * * * * * *$ \\
\hline & CSA & & & & & & & Laparoscopic & & & \\
\hline & & & & & & & & Robotic & & & \\
\hline \multirow[t]{2}{*}{ Yu et al [70] } & RENAL & 2018 & Int J Urol & Single & Matched-pair analysis & 375 & PN & Open & AKI or CKD progression & 3 & $* * * * * *$ \\
\hline & & & & & & & & Laparoscopic & & & \\
\hline Antonelli et al [71] & RENAL & 2019 & J Urol & Multiple & Randomized controlled trial & 149 & PN & Robotic & On-clamp excision & 1 & $3^{\mathrm{a}}$ \\
\hline \multirow[t]{2}{*}{ Qian et al [72] } & RENAL & 2019 & Urology & Single & Retrospective analysis & 225 & PN & Laparoscopic & Segmental artery clamp & 3 & $* * * * * * *$ \\
\hline & MAP & & & & & & & & & & \\
\hline \multirow[t]{3}{*}{ Ficarra et al [20] } & CSA & 2019 & BJU Int & Multiple & Retrospective analysis & 531 & PN & Open & Overall complications & 3 & ${ }^{* * * * * * * *}$ \\
\hline & SPARE & & & & & & & Laparoscopic & & & \\
\hline & & & & & & & & Robotic & & & \\
\hline \multicolumn{12}{|c|}{$\begin{array}{l}\text { ABC = Arterial Based Complexity; AKI = acute kidney injury; C-index = Centrality Index; } C K D=\text { chronic kidney disease; } C S=\text { collecting system; CSA = Contact Surface Area; DAP = Diameter-Axial-Polar; eGFR = estimatec } \\
\text { GFR; GFR = glomerular filtration rate; LE = level of evidence; MAP = Mayo Adhesive Probability; MIS = minimally invasive surgery; NePhRO = Nearness-Physical-Radius-Organization; OPN = open partial nephrectomy } \\
\text { PADUA = Preoperative Aspects and Dimensions Used for an Anatomical; PASS = Peritumoral Artery Scoring System; PN = partial nephrectomy; RAIV = Renal and Ischemia Volume; RENAL = Radius-Exophytic/Endophytic - } \\
\text { Nearness-Anterior/Posterior-Location; RN = radical nephrectomy; RPS = Renal Pelvic Score; RTII = Renal Tumor Invasion Index; SARR = Surgical Approach Renal Ranking; SPARE = Simplified PAdua REnal; SQ = study quality } \\
\text { UCS = urinary collecting system; ZII = Zero Ischemia Index; ZS = Zhongshan Score. } \\
\text { a LE according to the Jadad scale for randomized controlled trials. }\end{array}$} \\
\hline
\end{tabular}


Table 2 - Predictive values of nephrometry scores.

\begin{tabular}{|c|c|c|c|c|c|c|c|c|}
\hline Outcomes & $\tau^{2}$ & $\chi^{2}$ & df & $p$ value & $1^{2}(\%)$ & OR & $95 \% \mathrm{CI}$ & $p$ value \\
\hline \multicolumn{9}{|l|}{ RENAL } \\
\hline Minimally invasive surgery & 0.28 & 28.29 & 1 & 0.000 & 96.5 & 0.95 & $0.44,2.03$ & $0.896^{\mathrm{a}}$ \\
\hline PN vs $\mathrm{RN}^{\mathrm{b}}$ & - & - & - & - & - & 30.45 & $8.73,106.10$ & $<\mathbf{0 . 0 0 1}^{\mathrm{a}}$ \\
\hline On-clamp resection & 0.02 & 4.13 & 2 & 0.127 & 51.6 & 1.55 & $1.23,1.95$ & $<\mathbf{0 . 0 0 1}^{\mathrm{a}}$ \\
\hline \multirow[t]{2}{*}{ Warm ischemia time } & 0.04 & 5.04 & 2 & 0.082 & 60.3 & 1.53 & $1.13,2.06$ & $\mathrm{0.006}^{\mathrm{a}}$ \\
\hline & 0.05 & 1.32 & 1 & 0.251 & 24.1 & 9.29 & $5.37,16.06$ & $<0.001^{\mathrm{C}}$ \\
\hline \multirow[t]{2}{*}{ Overall complications } & 0.01 & 24.05 & 9 & 0.004 & 62.6 & 1.17 & $1.05,1.30$ & $0.002^{\mathrm{a}}$ \\
\hline & 0.00 & 2.87 & 6 & 0.825 & 0.0 & 2.75 & $1.80,4.23$ & $<0.001^{\mathrm{C}}$ \\
\hline \multirow{2}{*}{ Major complications } & 0.00 & 1.70 & 3 & 0.638 & 0.0 & 1.07 & $0.96,1.21$ & $0.212^{\mathrm{a}}$ \\
\hline & 0.00 & 3.03 & 4 & 0.553 & 0.0 & 3.55 & $2.00,6.28$ & $<0.001^{c}$ \\
\hline \multirow[t]{2}{*}{ Conversion to RN } & 0.00 & 0.01 & 1 & 0.940 & 0.0 & 1.40 & $1.14,1.73$ & $0.001^{\mathrm{a}}$ \\
\hline & 1.07 & 7.30 & 2 & 0.026 & 72.6 & 1.52 & $0.38,6.03$ & $0.551^{\mathrm{c}}$ \\
\hline \multirow{2}{*}{ Pelvicalyceal system entry/repair } & 0.02 & 2.02 & 1 & 0.155 & 50.5 & 1.53 & $1.15,2.04$ & $0.003^{a}$ \\
\hline & 1.72 & 4.63 & 1 & 0.031 & 78.4 & 6.42 & $0.83,49.78$ & $0.075^{\mathrm{c}}$ \\
\hline Urine leak ${ }^{\mathrm{b}}$ & - & - & - & - & - & 1.56 & $1.17,2.06$ & $\mathbf{0 . 0 0 2}^{\mathrm{a}}$ \\
\hline Malignancy & 1.44 & 4.09 & 1 & 0.043 & 75.6 & 3.49 & $0.54,22.63$ & $0.190^{c}$ \\
\hline New-onset CKD & 0.04 & 8.10 & 2 & 0.017 & 75.3 & 1.28 & $0.96,1.70$ & $0.086^{\mathrm{a}}$ \\
\hline \multirow[t]{2}{*}{ Renal function variation } & 0.00 & 0.56 & 1 & 0.455 & 0.0 & 1.28 & $1.07,1.53$ & $\mathbf{0 . 0 0 6}^{\mathrm{a}}$ \\
\hline & 0.14 & 1.46 & 1 & 0.228 & 31.3 & 5.64 & $2.22,14.32$ & $<\mathbf{0 . 0 0 1}^{\mathrm{C}}$ \\
\hline Trifecta achievement ${ }^{\mathrm{b}}$ & - & - & - & - & - & 0.77 & $0.34,1.70$ & $0.518^{\mathrm{c}}$ \\
\hline \multicolumn{9}{|l|}{ PADUA } \\
\hline PN vs $\mathrm{RN}^{\mathrm{b}}$ & - & - & - & - & - & 0.72 & $0.60,0.86$ & $<\mathbf{0 . 0 0 1}^{\mathrm{a}}$ \\
\hline On-clamp resection ${ }^{\mathrm{b}}$ & - & - & - & - & - & 1.53 & $1.23,1.90$ & $<\mathbf{0 . 0 0 1}^{\mathrm{a}}$ \\
\hline \multirow[t]{2}{*}{ Warm ischemia time } & 0.00 & 2.55 & 2 & 0.280 & 21.5 & 1.28 & $1.12,1.45$ & $<\mathbf{0 . 0 0 1}^{\mathrm{a}}$ \\
\hline & 0.00 & 2.63 & 4 & 0.621 & 0.0 & 2.93 & $2.05,4.20$ & $<\mathbf{0 . 0 0 1}^{\mathrm{c}}$ \\
\hline \multirow[t]{2}{*}{ Overall complications } & 0.00 & 7.48 & 5 & 0.187 & 33.1 & 1.34 & $1.20,1.49$ & $<\mathbf{0 . 0 0 1}^{\mathrm{a}}$ \\
\hline & 0.34 & 12.62 & 5 & 0.027 & 60.4 & 2.23 & $1.21,4.13$ & $0.010^{c}$ \\
\hline Major complications & - & - & - & - & - & 1.95 & $1.29,2.94$ & $\mathbf{0 . 0 0 1}^{\mathrm{a}, \mathrm{b}}$ \\
\hline & 0.00 & 0.60 & 2 & 0.742 & 0.0 & 2.39 & $1.36,4.20$ & $0.002^{c}$ \\
\hline Pelvicalyceal system entry/repair ${ }^{b}$ & - & - & - & - & - & 1.41 & $1.18,1.67$ & $<\mathbf{0 . 0 0 1}^{\mathrm{a}}$ \\
\hline & 0.00 & 0.13 & 1 & 0.722 & 0.0 & 3.27 & $1.96,5.46$ & $<\mathbf{0 . 0 0 1}^{\mathrm{c}}$ \\
\hline Renal function variation ${ }^{\mathrm{b}}$ & - & - & - & - & - & 0.60 & $0.36,1.00$ & $0.050^{\mathrm{a}}$ \\
\hline Trifecta achievement $\mathrm{t}^{\mathrm{b}}$ & - & - & - & - & - & 0.88 & $0.14,5.18$ & $0.888^{\mathrm{c}}$ \\
\hline C-index & & & & & & & & \\
\hline Overall complications ${ }^{\mathrm{b}}$ & - & - & - & - & - & 0.63 & $0.49,0.81$ & $<\mathbf{0 . 0 0 1}^{\mathrm{a}}$ \\
\hline DAP & & & & & 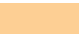 & & & \\
\hline Warm ischemia time ${ }^{\mathrm{b}}$ & - & - & - & - & - & 1.74 & $1.37,2.20$ & $<\mathbf{0 . 0 0 1}^{\mathrm{a}}$ \\
\hline Renal function variation ${ }^{\mathrm{b}}$ & - & - & - & - & - & 1.29 & $1.04,1.59$ & $0.018^{\mathrm{a}}$ \\
\hline $\mathrm{NePhRO}$ & & & & & & & & \\
\hline Overall complications ${ }^{\mathrm{b}}$ & - & - & - & - & - & 1.20 & $1.03,1.44$ & $0.020^{a}$ \\
\hline & - & - & - & - & - & 3.24 & $1.15,9.12$ & $0.026^{c}$ \\
\hline RTII & & & & & & & & \\
\hline PN vs $\mathrm{RN}^{\mathrm{b}}$ & - & - & - & - & - & 0.55 & $0.19,1.58$ & $0.267^{\mathrm{a}}$ \\
\hline 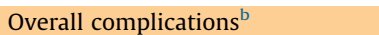 & - & - & - & - & - & 2.91 & $1.52,5.52$ & $\mathbf{0 . 0 0 1}^{\mathrm{a}}$ \\
\hline RPS & & & & & & & & \\
\hline Overall complications & 0.00 & 0.48 & 1 & 0.002 & 0.0 & 34.25 & $13.89,84.43$ & $<\mathbf{0 . 0 0 1}^{\mathrm{a}}$ \\
\hline Major complications & 0.00 & 0.49 & 1 & 0.483 & 0.0 & 34.25 & $13.89,84.43$ & $<\mathbf{0 . 0 0 1}^{\mathrm{a}}$ \\
\hline & & & & & & 1.11 & $0.30,4.10$ & $0.876^{c}$ \\
\hline Urine leak & 0.00 & 0.49 & 1 & 0.483 & 0.0 & 34.12 & $13.87,83.93$ & $<\mathbf{0 . 0 0 1}^{\mathrm{a}}$ \\
\hline SARR & & & & & & & & \\
\hline PN vs $\mathrm{RN}^{\mathrm{b}}$ & - & - & - & - & - & 39.53 & $10.55,148.13$ & $<0.0011^{a}$ \\
\hline Overall complications ${ }^{\mathrm{b}}$ & - & - & - & - & - & 1.39 & $0.36,5.31$ & $0.631^{c}$ \\
\hline CSA & & & & & & & & \\
\hline Warm ischemia time ${ }^{b}$ & - & - & - & - & - & 3.51 & $1.24,9.94$ & $0.018^{\mathrm{c}}$ \\
\hline Overall complications & 0.00 & 9.25 & 1 & 0.002 & 89.2 & 1.05 & $0.98,1.13$ & $0.152^{\mathrm{a}}$ \\
\hline & - & - & - & - & - & 4.08 & $1.40,11.88$ & $\mathbf{0 . 0 1 0}$ \\
\hline MAP & & & & & & & & \\
\hline Minimally invasive surgery ${ }^{\mathrm{b}}$ & - & - & - & - & - & 1.04 & $0.81,1.33$ & $0.760^{\mathrm{a}}$ \\
\hline Adhesive perinephric fat & 0.07 & 2.73 & 1 & 0.098 & 63.4 & 1.98 & $1.23,3.18$ & $\mathbf{0 . 0 0 5}^{\mathrm{a}}$ \\
\hline On-clamp resection ${ }^{\mathrm{b}}$ & - & - & - & - & - & 3.91 & $2.18,6.99$ & $<\mathbf{0 . 0 0 1}^{\mathrm{a}}$ \\
\hline Overall complications ${ }^{\mathrm{b}}$ & - & - & - & - & - & 1.05 & $0.86,1.28$ & $0.631^{\mathrm{a}}$ \\
\hline & - & - & - & - & - & 1.32 & $0.56,3.08$ & $0.522^{c}$ \\
\hline Major complications ${ }^{\mathrm{b}}$ & - & - & - & - & - & 1.11 & $0.81,1.52$ & $0.516^{\mathrm{a}}$ \\
\hline & - & - & - & - & - & 1.10 & $0.30,4.09$ & $0.876^{\mathrm{c}}$ \\
\hline Conversion to $\mathrm{RN}^{\mathrm{b}}$ & - & - & - & - & - & 7.66 & $3.10,18.94$ & $<0.001^{\mathrm{a}}$ \\
\hline & - & - & - & - & - & 3.29 & $1.39,7.77$ & $0.007^{c}$ \\
\hline Trifecta achievement $^{\mathrm{b}}$ & - & - & - & - & - & 0.79 & $0.38,1.60$ & $0.515^{\mathrm{c}}$ \\
\hline RAIV & & & & & & & & \\
\hline Renal function variation ${ }^{\mathrm{b}}$ & - & - & - & - & - & 1.11 & $1.00,1.23$ & $0.052^{\mathrm{a}}$ \\
\hline
\end{tabular}




\begin{tabular}{|c|c|c|c|c|c|c|c|c|}
\hline Outcomes & $\tau^{2}$ & $x^{2}$ & df & $p$ value & $1^{2}(\%)$ & OR & $95 \%$ CI & $p$ value \\
\hline \multicolumn{9}{|l|}{$\mathrm{ABC}$} \\
\hline On-clamp resection ${ }^{\mathrm{b}}$ & - & - & - & - & - & 1.84 & $1.31,2.57$ & $<\mathbf{0 . 0 0 1}^{\mathrm{a}}$ \\
\hline Warm ischemia time ${ }^{b}$ & - & - & - & - & - & 1.25 & $0.95,1.62$ & $0.098^{\mathrm{a}}$ \\
\hline \multirow[t]{2}{*}{ Overall complications $^{\mathrm{b}}$} & - & - & - & - & - & 1.31 & $0.88,1.94$ & $0.181^{\mathrm{a}}$ \\
\hline & - & - & - & - & - & 1.38 & $0.93,2.04$ & $0.108^{c}$ \\
\hline Pelvicalyceal system entry/repair ${ }^{\mathrm{b}}$ & - & - & - & - & - & 1.85 & $1.37,2.49$ & $<\mathbf{0 . 0 0 1}^{\mathrm{a}}$ \\
\hline Renal function variation ${ }^{\mathrm{b}}$ & - & - & - & - & - & 3.98 & $0.09,164.91$ & $0.467^{\mathrm{c}}$ \\
\hline \multicolumn{9}{|l|}{ ZS } \\
\hline Overall complications ${ }^{\mathrm{b}}$ & - & - & - & - & - & 3.70 & $1.71,8.08$ & $0.001^{c}$ \\
\hline \multicolumn{9}{|l|}{ ZII } \\
\hline Overall complications ${ }^{\mathrm{b}}$ & - & - & - & - & - & 1.26 & $1.04,1.52$ & $0.019^{a}$ \\
\hline \multicolumn{9}{|l|}{ PASS } \\
\hline \multicolumn{9}{|l|}{ Renal function variation ${ }^{\mathrm{b}}$} \\
\hline \multicolumn{9}{|l|}{ SPARE } \\
\hline Overall complications ${ }^{\mathrm{b}}$ & - & - & - & - & - & 1.20 & $1.10,1.30$ & $<\mathbf{0 . 0 0 1}^{\mathrm{a}}$ \\
\hline \multicolumn{9}{|c|}{$\begin{array}{l}\text { ABC = Arterial Based Complexity; } \mathrm{CI}= \\
\mathrm{DAP}=\text { Diameter-Axial-Polar; } \mathrm{df}=\text { degrees } \\
\text { PADUA = Preoperative Aspects and Dimen } \\
\text { Ischemia Volume; RENAL = Radius-Exop } \\
\text { = Renal Tumor Invasion Index; SARR = Su } \\
\text { Bold } p \text { values indicate statistical significa } \\
\text { a Nephrometry score value: continuous. } \\
\text { b Pooled analysis not possible. } \\
\text { c Nephrometry score value: categorical. }\end{array}$} \\
\hline
\end{tabular}

independent predictor of prolonged WIT. Similarly, the pooled analysis showed the PADUA score to predict prolonged WIT as a continuous variable (OR: 1.28 ; $95 \% \mathrm{CI}$ : 1.12 , $1.45 ; p<0.001)[33,54,58]$ and a categorical (high complexity) variable (OR: 2.93; 95\% CI: $2.05,4.20 ; p<0.001$ ) $[11,30,33,54,55]$. Only one study was reported as continuous on DAP [49] and ABC [59] and as categorical on CSA [11], with DAP and CSA found to be independent predictors of WIT ( $p<0.01$; Table 2 and the Supplementary material).

\subsubsection{Overall complications}

Continuous (OR: 1.17; 95\% CI: 1.05, 1.30; $p=0.002$ ) and high-complexity (OR: 2.75; 95\% CI: 1.80, 4.23; $p<0.001$ ) RENAL scores were found to be independent predictors of overall complications. In addition, the PADUA score was related to complications as continuous (OR: $1.34 ; 95 \% \mathrm{CI}$ : $1.20,1.49 ; p<0.001$ ) and categorical (OR: 2.23 ; 95\% CI: 1.21 , 4.13; $p<0.002$ ) value. A cumulative analysis was not possible for the NePhRO score [49], Zhongshan score [15], ZII [17], and SPARE [20], which was shown to be related to overall complications. CSA was correlated to overall complications as a categorical variable $(p=0.010)$ [11] but not as a continuous one $[20,69]$. C-index was not a predictor of overall complications (OR: 0.63 ; 95\% CI: 0.49, $0.81 ; p<$ 0.001 ; Table 2 and the Supplementary material) (Fig. 2).

\subsubsection{Major complications}

Both RENAL high complexity (OR: 3.55; 95\% CI: 2.00, 6.28; $p<0.001$ ) $[9,37,42,53,66]$ and PADUA categorical (OR: 2.39; 95\% CI: 1.36, 4.20; $p=0.002$ ) [42,54,65] were independent predictors of major complications. RPS was strongly related to the incidence of major complications (OR: $34.25 ; 95 \% \mathrm{CI}$ : 13.89, 84.43; $p<0.001)[9,43]$. A pooled analysis was not feasible for PADUA as well as for MAP as continuous variables because only one study reported these data for each nephrometry score $[50,65]$. Only PADUA was found to be a predictor of major complications (Table 2 and the Supplementary material).

\subsubsection{Conversion to $R N$}

Continuous RENAL score was an independent predictor of conversion to RN (OR: 1.40; 95\% CI: $1.14,1.73 ; p=0.001$ ) $[65,66]$, but this was not the case for high-complexity RENAL score $(p=0.551)$. Only one study reported about MAP as a predictor of conversion to RN both as continuous $(p<0.001)$ and as categorical $(p=0.007$; Table 2 and the Supplementary material).

\subsubsection{Pelvicalyceal system entry/repair}

Only two studies per nephrometry score were available regarding this outcome $[30,35,54,59]$. RENAL as a continuous variable was directly related to pelvicalyceal system entry/repair (OR: 1.53; 95\% CI: 1.15, 2.04; $p=0.003$ ) $[35,59]$. This was not the case for high-complexity RENAL score, whereas high-complexity PADUA score was shown to be an independent predictor of pelvicalyceal effraction (OR: 3.27; 95\% CI: 1.96, 5.46; $p<0.001$ ) [30,54]. Only one study assessed $A B C$, which was a strong predictor of pelvicalyceal system entry/repair $(p<0.001$; Table 2 and the Supplementary material).

\subsubsection{Urine leak}

RPS as a continuous value was demonstrated to predict the risk of urine leak strongly (OR: 34.12; 95\% CI: 13.87, 83.93; $p<0.001)[9,44]$. The only other nephrometry score evaluated regarding this outcome was the RENAL score, which 
was shown to predict urine leak too $(p=0.002$; Table 2 and the Supplementary material) [36].

\subsubsection{Malignant histology}

The categorical RENAL score was the only score to be assessed as a predictor of malignancy, but it was not found to be predictive ( $p=0.190$; Table 2 and the Supplementary material) $[39,47]$. A subanalysis of its single components showed that $N=3$ (OR: $1.98 ; 95 \% \mathrm{CI}: 1.18,3.34 ; p=0.010$ ) was an independent predictor of histological malignancy $[31,43,64]$. In only one study $R=3$ was assessed, which was shown to be a predictor of malignant histology and highgrade tumor (OR: $4.05 ; 95 \% \mathrm{CI}: 1.43,11.39 ; p=0.008$ and $\mathrm{OR}$ : 3.89; 95\% CI: 2.12, 7.11; $p<0.001$, respectively) [31]. RENAL $E=2$ was an independent predictor of high-grade tumor (OR: 1.67; 95\% CI: 1.07, 2.62; $p=0.024$; Fig. 3) [31,43,64].

\subsubsection{Postoperative renal function}

Meta-analysis of new-onset CKD was feasible for the RENAL score only, but it did not predict it ( $p=0.086$; Table 2 and the Supplementary material) $[38,41,70]$. In terms of renal function variation, continuous $[18,70]$ and categorical RENAL scores [19,63] were independent predictors of an estimated glomerular filtration rate (eGFR) increase (OR: 1.28; $95 \% \mathrm{CI}: 1.07,1.53 ; p=0.006$, and OR: $5.64 ; 95 \% \mathrm{CI}: 2.22$, $14.32 ; p<0.001$, respectively). A pooled analysis was not feasible for the other nephrometry scores because only one study per nephrometry score was available. The DAP score $(p=0.018)$ [49] and PASS $(p=0.02)$ [18] were independent predictors of renal function variation. PADUA $(p=0.05)[52]$ and RAIV $(p=0.052)$ [18] demonstrated a sort of correlation with renal function variation but they did not achieved the usual levels of statistical significance. (Table 2 and Fig. 4).

\subsubsection{Trifecta achievement}

None of the nephrometry scores included was demonstrated to be an effective predictor of trifecta achievement (Table 2 and the Supplementary material).

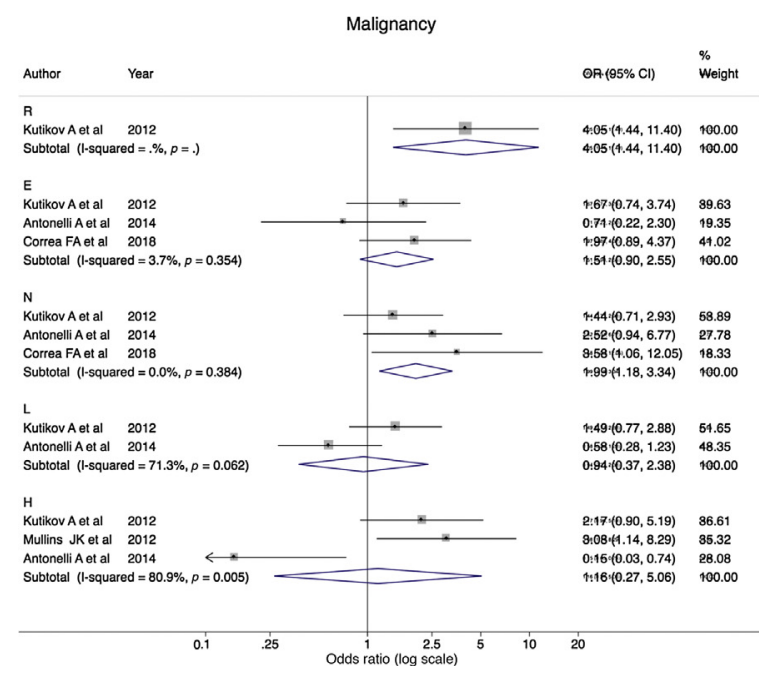

\subsection{Discussion}

Herein, we present the first systematic review and metaanalysis assessing the predictive role of nephrometry scores in NSS. Our findings raise some interesting points of discussion.

Since their introduction in 2009 [2,3], the aim of these tools was to facilitate preoperative planning, surgical decision making, and counseling. Our analysis found nephrometry scores to be a predictor of surgical strategy. Nevertheless, this was not the case regarding the decision to perform a MIS. Previous literature had suggested that patients with more complex renal masses were more likely to have an open PN [36]. Sharma et al [52] found that patients with a higher RENAL score were more likely to undergo an open procedure as well. On the contrary, the presence of adhesive perinephric fat did not influence the decision making. Today, this paradigm is changing, and MIS, especially robotic surgery, has been shown to be safe and feasible even for large and complex renal tumors. Data from the ROSULA Collaborative Group demonstrated robotic PN to be feasible for large renal masses, maximizing kidney function without compromising on oncological outcomes [73]. Despite the encouraging data regarding NSS for T1b-T2 tumors [74], RN is recommended when PN is not feasible [1], and our data demonstrated the role of the nephrometry score during preoperative planning. Indeed, higher RENAL and PADUA scores increased the chances of opting for RN rather than PN. Tannus et al [10] developed an "ad hoc" nephrometry score, the SARR, which assessed the relationship between tumor complexity and the odds of RN. The authors evaluated the computed tomography and magnetic resonance images of 257 patients, showing that patients with higher SARR had almost 39-fold odds to undergo RN compared with those with smaller scores [37]. This is the only study in the literature on this score.

We also assessed the predictive role of the scores regarding hilar management during tumor resection. RENAL,

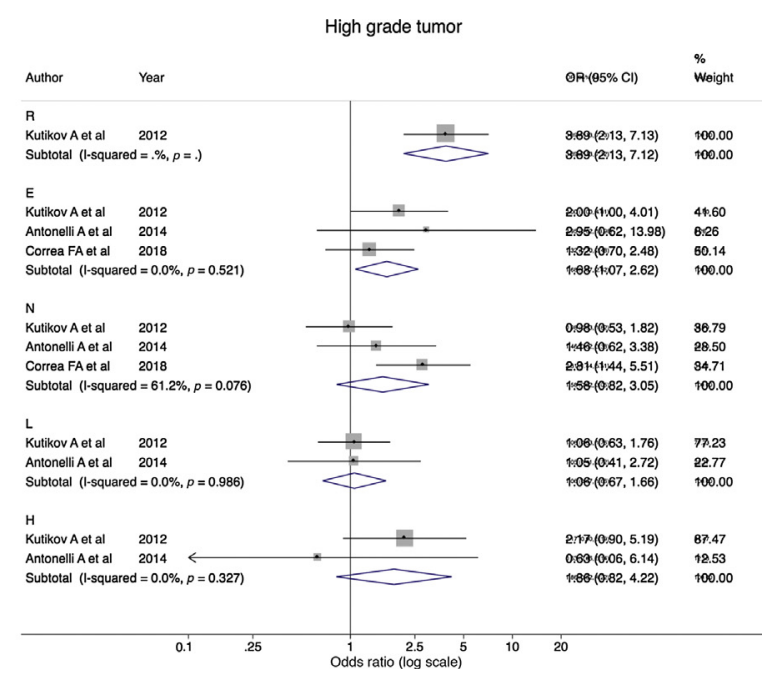

Fig. 3 - Histology predictive value of RENAL components. $\mathrm{CI}=$ confidence interval; OR = odds ratio; RENAL = Radius-Exophytic/Endophytic-NearnessAnterior/Posterior-Location. 

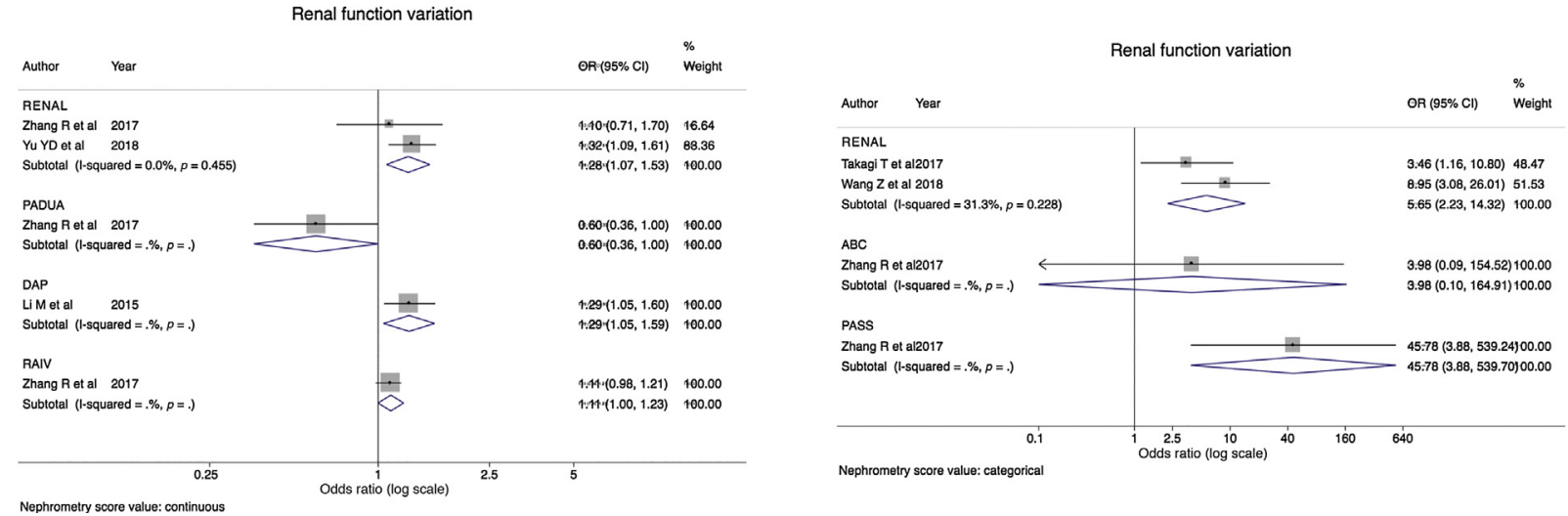

Fig. 4 - Predictive value of Nephrometry score for renal function variation. $\mathrm{ABC}=$ Arterial Based Complexity; $\mathrm{CI}=$ confidence interval; $\mathrm{DAP}=\mathrm{Diameter}-$ Axial-Polar; OR = odds ratio; PADUA = Preoperative Aspects and Dimensions Used for an Anatomical; PASS = Peritumoral Artery Scoring System; RAIV = Renal and Ischemia Volume; RENAL = Radius-Exophytic/Endophytic-Nearness-Anterior/Posterior-Location.

PADUA, and ABC were predictors of hilar clamping, but the MAP score seemed to be the strongest one [72]. Data from a new randomized trial suggested a higher RENAL score to be a predictor of the transition from an off-clamp to an onclamp robotic PN [71]. Recently, the CLAMP score has been developed to evaluate patients suitable for selective artery clamping. This is a 3D imaging-based tool that allows evaluation of the opportunity to perform e selective artery clamp, stratifying patients according to the vascular anatomy [19]. The use of scoring systems to predict the clamping technique remains poorly investigated.

Regarding surgical outcomes, a higher nephrometry score directly influenced WIT, which is a surrogate of tumor complexity [75]. Indeed, a pooled analysis of RENAL and PADUA demonstrated an increased probability of longer WIT. These data were already achieved by a large multicenter study on 227 patients. In this report, the authors concluded that patients with high-complexity RENAL and PADUA scores had 5.7- and 2.6-fold higher rates of longer WIT, respectively [54]. Besides RENAL and PADUA, only CSA, $\mathrm{DAP}$, and $\mathrm{ABC}$ were investigated regarding the duration of ischemia time $[11,49,59]$. The $A B C$ score was the only one unrelated to WIT, and this could be a consequence of its design that accounts only for tumor location, disregarding other data such as diameter, degree of depth, and longitudinal position. Interestingly, Kriegmair et al [59] evaluated a modified version of the $\mathrm{ABC}$ score that also included tumor diameter. The authors found that the inclusion of this parameter made the $A B C$ an independent predictor of WIT. These data suggest that tumor dimension is one of the main parameters to consider during nephrometry score development [62].

Overall and major complications were the most investigated outcomes among the nephrometry scores. The first-generation nephrometry scores showed once again their predictive role of overall complications as both continuous and categorical variables. Kriegmair et al [76] achieved the same result in a large cohort series comparing four different scores. Interestingly, the authors analyzed NePhRO score and C-index, with the first related to complications $(p=0.011)$. These data reflected our results showing the inferiority of C-index compared with the others. In addition, except for C-index and MAP score, all the nephrometry scores appeared to be potentially independent predictors of overall and major complications. Nevertheless, RPS was underlined to be superior with 34.25-fold chances of complications for patients with a higher score. Particularly, RPS assessed the renal pelvic anatomic complexity and seemed to be strictly related to urine leak incidence and duration. Tomaszewski et al [9] speculated that the reason could be the interpretation of RPS as a surrogate of renal pelvis volume and pressure. Thus, intrarenal pelvis might present higher inner pressure, increasing the risk of rupture during tumor resection and yielding to delayed healing of urine leak. The urine leak could be a consequence of more complex tumor resection. In fact, higher nephrometry scores and complexity were related to pelvicalyceal system entry/repair. Potretzke et al [77] conducted a review of studies on urinary fistula after robotic PN, and found that pelvicalyceal system entry and tumor size were related to the development of urine leak. These two parameters could be interpreted as a reflection of tumor complexity, which could be the reason for urinary fistula incidence. Our data reflect these findings, and RENAL score and PADUA complexity were shown to be linked to pelvicalyceal system entry/repair. Potentially, a higher ABC score could indicate the risk of urinary tract effraction/repair, but only one study in our analysis reported this outcome [59].

Another consequence of tumor complexity is the risk of intraoperative conversion to RN. In our review, few analyses reported these data $[37,59,65,66]$, and pooled estimation underlined the RENAL score correlation with conversion rate. Nevertheless, the MAP score overcame the RENAL score. Indeed, patients with a higher MAP score and a higher MAP risk had, respectively, 7.66- and 3.29-fold higher risks to be converted to RN. It might be speculated that a higher MAP score could be a consequence of advanced disease, prompting the surgeon to convert the case to a radical one [78]. 
The potential role of nephrometry scores to predict malignancy and tumor grade was postulated by Kutikov et al [31]. The authors evaluated each RENAL score component on a cohort of 525 patients and found that $R=3, E=2$, and $L=3$ were predictors of malignant histology and high-grade tumors. Equally, we found $R=3, E=2$, and hilar location as predictors of malignancy. Correa et al [64] hypothesized that tumor growth within the inner renal environment could promote its progression, explaining the major aggressiveness of hilar and endophytic masses. Notably, high-stage, hilarlocated renal tumors, especially clear cell carcinomas, showed a higher expression of GLUT5, which is related to glucose metabolism and neoplasm growth [79].

In terms of functional outcomes, the RENAL score was not an independent predictor of new-onset CKD. On the contrary, it was found to be linked to postoperative renal function variation, as well as PADUA and PASS. The latter, which is a $3 \mathrm{D}$ rendering-based score assessing peritumoral artery volume, was demonstrated to be the strongest predictor of eGFR variation. The authors found that tumors with higher PASS scores were more likely to have higher RENAL scores too. Consequently, resection could be more difficult, requiring longer ischemia time and larger healthy parenchyma removal [80]. The aforementioned reasons could unfold the strong relationship between PASS and renal function variation. Zhang et al [16] developed a mathematical score to evaluate the acute ipsilateral renal dysfunction after PN: the Spectrum score. This interesting score is based on the following formula: (observed peak $\mathrm{SCr}-$ $\left.\mathrm{SCr}_{\text {ideal-peak }}\right) /\left(\mathrm{SCr}_{\text {worstcase}}{ }^{-}\right.$peak $-\mathrm{SCr}_{\text {ideal }}{ }^{-}$peak $)$; its modified version, proposed by Lee et al [81], quantified acute ipsilateral renal dysfunction and renal recovery after PN (beta $-0.515, p<0.001$ ). This novel tool could better forecast renal function variation, but available data are still weak to consider it as an effective predictor.

Overall, RENAL and PADUA seem to represent the best tools to report complexity and prediction of morbidity. Despite other nephrometry scores being promising, their role in predicting specific outcomes does not outperform these first-generation scores.

This study presents limitations. The reports included were all retrospective and of intermediate quality, and the only randomized trial did not address the topic specifically [71]. Moreover, a cumulative analysis was possible only for a limited number of scores and a limited number of outcomes. Therefore, a narrative review was adopted to summarize some of the outcomes. In addition, it was not possible to account for open, laparoscopic, or robotic procedures, so the results might be reliable for one technique but not for the other. The interobserver variability could have influenced the results, but these data were not accountable again. Last, most of the literature available comes from repeat publications from the same working group; therefore, one can argue that some data could have been assessed within the same cohort, translating into an additional bias. Notwithstanding these limitations, the main strength of this study is its design as a systematic review and meta-analysis that makes it depart from the previous descriptive ones.

\section{Conclusions}

The literature on nephrometry scoring systems is sparse, and it is mostly focused on RENAL and PADUA. These two scores are easy to calculate, and they carry a good correlation with most of the outcomes for which they have been assessed. RPS, SARR, and PASS can offer a better predictive value for pelvicalyceal entry/repair and urine leak, surgical approach, and renal function variation, respectively. Nevertheless, the implementation of other nephrometry scores based on mathematical models is limited by their complexity and lack of evidence supporting their predictive value. Up to date, the RENAL and PADUA scores can be regarded as the standards for reporting complexity and prediction of morbidity, whereas other newer tools did not show better performance than the first-generation ones. The present findings can aid in further research effort in this field and foster the development of better predictive tools.

Author contributions: Riccardo Autorino had full access to all the data in the study and takes responsibility for the integrity of the data and the accuracy of the data analysis.

Study concept and design: Veccia, Autorino.

Acquisition of data: Veccia, Antonelli.

Analysis and interpretation of data: Veccia, Autorino.

Drafting of the manuscript: Veccia, Autorino.

Critical revision of the manuscript for important intellectual content: Autorino, Veccia, Antonelli, Uzzo, Novara, Kutikov, Ficarra, Simeone, Mirone, Hampton, Derweesh, Porpiglia.

Statistical analysis: Veccia. Obtaining funding: None.

Administrative, technical, or material support: None.

Supervision: Autorino, Antonelli, Uzzo, Novara, Kutikov, Ficarra, Simeone, Mirone, Hampton, Derweesh, Porpiglia.

Other: None.

Financial disclosures: Riccardo Autorino certifies that all conflicts of interest, including specific financial interests and relationships and affiliations relevant to the subject matter or materials discussed in the manuscript (eg, employment/affiliation, grants or funding, consultancies, honoraria, stock ownership or options, expert testimony, royalties, or patents filed, received, or pending), are the following: Dr. Veccia is an Italian Society of Urology-American Urological Association (SIU-AUA) research fellow. Funding for his fellowship is also provided by the VCU Urology Research Fund.

Funding/Support and role of the sponsor: None.

\section{Appendix A. Supplementary data}

Supplementary material related to this article can be found, in the online version, at doi:https://doi.org/10. 1016/j.euf.2019.11.004.

\section{References}

[1] Ljungberg B, Albiges L, Abu-Ghanem Y, et al. European Association of Urology guidelines on renal cell carcinoma: the 2019 update. Eur Urol 2019;75:799-810. 
[2] Kutikov A, Uzzo RG. The R.E.N.A.L. nephrometry score: a comprehensive standardized system for quantitating renal tumor size, location and depth. J Urol 2009;182:844-53.

[3] Ficarra V, Novara G, Secco S, et al. Preoperative aspects and dimensions used for an anatomical (PADUA) classification of renal tumours in patients who are candidates for nephron-sparing surgery. Eur Urol 2009;56:786-93.

[4] Klatte T, Ficarra V, Gratzke C, et al. A literature review of renal surgical anatomy and surgical strategies for partial nephrectomy. Eur Urol 2015;68:980-92.

[5] Simmons MN, Ching CB, Samplaski MK, Park CH, Gill IS. Kidney tumor location measurement using the $\mathrm{C}$ index method. J Urol 2010;183:1708-13.

[6] Simmons MN, Hillyer SP, Lee BH, Fergany AF, Kaouk J, Campbell SC. Diameter-axial-polar nephrometry: integration and optimization of R.E.N.A.L. and centrality index scoring systems. J Urol 2012;188:384-90.

[7] Hakky TS, Baumgarten AS, Allen B, et al. Zonal NePhRO scoring system: a superior renal tumor complexity classification model. Clin Genitourin Cancer 2014;12:e13-8.

[8] Nisen H, Ruutu M, Glücker E, Visapää H, Taari K. Renal tumour invasion index as a novel anatomical classification predicting urological complications after partial nephrectomy. Scand J Urol 2014;48:41-51.

[9] Tomaszewski JJ, Cung B, Smaldone MC, et al. Renal pelvic anatomy is associated with incidence, grade, and need for intervention for urine leak following partial nephrectomy. Eur Urol 2014;66:949-55.

[10] Tannus M, Goldman SM, Andreoni C. Practical and intuitive surgical approach renal ranking to predict outcomes in the management of renal tumors: a novel score tool. J Endourol 2014;28:487-92.

[11] Leslie S, Gill IS, de Castro Abreu AL, et al. Renal tumor contact surface area: a novel parameter for predicting complexity and outcomes of partial nephrectomy. Eur Urol 2014;66:884-93.

[12] Davidiuk AJ, Parker AS, Thomas CS, et al. Mayo Adhesive Probability score: an accurate image-based scoring system to predict adherent perinephric fat in partial nephrectomy. Eur Urol 2014;66:1165-71.

[13] Shin TY, Komninos C, Kim DW, et al. A novel mathematical model to predict the severity of postoperative functional reduction before partial nephrectomy: the importance of calculating resected and ischemic volume. J Urol 2015;193:423-9.

[14] Spaliviero M, Poon BY, Karlo CA, et al. An Arterial Based Complexity $(A B C)$ scoring system to assess the morbidity profile of partial nephrectomy. Eur Urol 2016;69:72-9.

[15] Zhou L, Guo J, Wang H, Wang G. The Zhongshan score: a novel and simple anatomic classification system to predict perioperative outcomes of nephron-sparing surgery. Medicine (Baltimore) 2015;94: e506.

[16] Zhang Z, Zhao J, Dong W, et al. Acute ipsilateral renal dysfunction after partial nephrectomy in patients with a contralateral kidney: spectrum score to unmask ischemic injury. Eur Urol 2016;70:692-8.

[17] Li Y, Zhou L, Bian T, et al. The Zero Ischemia Index (ZII): a novel criterion for predicting complexity and outcomes of off-clamp partial nephrectomy. World J Urol 2017;35:1095-102.

[18] Zhang R, Wu G, Huang J, et al. Peritumoral Artery Scoring System: a novel scoring system to predict renal function outcome after laparoscopic partial nephrectomy. Sci Rep 2017;7:2853.

[19] Wang Y, Chen C, Qin C, et al. The C.L.A.M.P. nephrometry score: a system for preoperative assessment of laparoscopic partial nephrectomy with segmental renal artery clamping. Sci Rep 2018;8:9717.

[20] Ficarra V, Porpiglia F, Crestani A, et al. The Simplified PAdua REnal (SPARE) nephrometry system: a novel classification of parenchymal renal tumors suitable for partial nephrectomy. BJU Int. In press. https://doi.org/10.1111/bju.14772.
[21] Bier S, Aufderklamm S, Todenhöfer T, et al. Prediction of postoperative risks in laparoscopic partial nephrectomy using RENAL, Mayo Adhesive Probability and Renal Pelvic Score. Anticancer Res 2017;37:1369-73.

[22] Antonelli A, Veccia A, Sandri M, et al. External validation of the Arterial-Based Complexity score and first head-to-head comparison with the R.E.N.A.L. and PADUA scores and C-index. Clin Genitourin Cancer 2018;16:e595-604.

[23] Moher D, Liberati A, Tetzlaff J, Altman DG, PRISMA Group. Preferred reporting items for systematic reviews and meta-analyses: the PRISMA statement. Int J Surg 2010;8:336-41.

[24] Moher D, Shamseer L, Clarke M, et al. Preferred reporting items for systematic review and meta-analysis protocols (PRISMA-P) 2015 statement. Syst Rev (4):2015:1.

[25] Howick J, Chalmers I, Glasziou P, et al. Explanation of the 2011 Oxford Centre for Evidence-Based Medicine (OCEBM) levels of evidence (background document). Oxford Centre for EvidenceBased Medicine. http://www.cebm.net/index.aspx?o=5653.

[26] Wells GA, Shea B, O'Connell D, et al. The Newcastle-Ottawa Scale (NOS) for assessing the quality of nonrandomized studies in metaanalyses. Ottawa Hospital Research Institute 2013;1-4.

[27] Jadad AR, Moore RA, Carroll D, et al. Assessing the quality of reports of randomized clinical trials: is blinding necessary? Control Clin Trials 1996; 17:1-12.

[28] Ades AE, Lu G, Higgins JP. The interpretation of random-effects metaanalysis in decision models. Med Decis Making 2005;25:646-54.

[29] Egger M, Smith GD, Schneider M, Minder C. Bias in meta-analysis detected by a simple, graphical test. BMJ 1997;315:629-34.

[30] Mottrie A, Schatteman P, De Wil P, De Troyer B, Novara G, Ficarra V. Validation of the preoperative aspects and dimensions used for an anatomical (PADUA) score in a robot-assisted partial nephrectomy series. World J Urol 2013;31:799-804.

[31] Kutikov A, Smaldone MC, Egleston BL, et al. Anatomic features of enhancing renal masses predict malignant and high-grade pathology: a preoperative nomogram using the RENAL nephrometry score. Eur Urol 2011;60:241-8.

[32] Simhan J, Smaldone MC, Tsai KJ, et al. Objective measures of renal mass anatomic complexity predict rates of major complications following partial nephrectomy. Eur Urol 2011;60:724-30.

[33] Ficarra V, Bhayani S, Porter J, et al. Predictors of warm ischemia time and perioperative complications in a multicenter, international series of robot-assisted partial nephrectomy. Eur Urol 2012;61:395-402.

[34] Liu ZW, Olweny EO, Yin G, et al. Prediction of perioperative outcomes following minimally invasive partial nephrectomy: role of the R.E.N.A.L nephrometry score. World J Urol 2013;31:1183-9.

[35] Mayer WA, Godoy G, Choi JM, Goh AC, Bian SX, Link RE. Higher RENAL nephrometry score is predictive of longer warm ischemia time and collecting system entry during laparoscopic and roboticassisted partial nephrectomy. Urology 2012;79:1052-6.

[36] Stroup SP, Palazzi K, Kopp RP, et al. RENAL nephrometry score is associated with operative approach for partial nephrectomy and urine leak. Urology 2012;80:151-6.

[37] Long JA, Arnoux V, Fiard G, et al. External validation of the RENAL nephrometry score in renal tumours treated by partial nephrectomy. BJU Int 2013;111:233-9.

[38] Kopp RP, Mehrazin R, Palazzi K, Bazzi WM, Patterson AL, Derweesh IH. Factors affecting renal function after open partial nephrectomya comparison of clampless and clamped warm ischemic technique. Urology 2012;80:865-70.

[39] Mullins JK, Kaouk JH, Bhayani S, et al. Tumor complexity predicts malignant disease for small renal masses. J Urol 2012;188:2072-6.

[40] Tanagho YS, Kaouk JH, Allaf ME, et al. Perioperative complications of robot-assisted partial nephrectomy: analysis of 886 patients at 5 United States centers. Urology 2013;81:573-9. 
[41] Mehrazin R, Palazzi KL, Kopp RP, et al. Impact of tumour morphology on renal function decline after partial nephrectomy. BJU Int 2013;111:E374-82.

[42] Krane LS, Manny TB, Mufarrij PW, Hemal AK. Does experience in creating a robot-assisted partial nephrectomy (RAPN) programme in an academic centre impact outcomes or complication rate? BJU Int 2013;112:207-15.

[43] Antonelli A, Furlan M, Sandri M, et al. The R.E.N.A.L. nephrometric nomogram cannot accurately predict malignancy or aggressiveness of small renal masses amenable to partial nephrectomy. Clin Genitourin Cancer 2014;12:366-72.

[44] Tomaszewski JJ, Smaldone MC, Cung B, et al. Internal validation of the renal pelvic score: a novel marker of renal pelvic anatomy that predicts urine leak after partial nephrectomy. Urology 2014;84:351-7.

[45] Reddy UD, Pillai R, Parker RA, et al. Prediction of complications after partial nephrectomy by RENAL nephrometry score. Ann R Coll Surg Engl 2014;96:475-9.

[46] Kocher NJ, Kunchala S, Reynolds C, et al. Adherent perinephric fat at minimally invasive partial nephrectomy is associated with adverse peri-operative outcomes and malignant renal histology. BJU Int 2016;117:636-41.

[47] Ball MW, Gorin MA, Bhayani SB, et al. Preoperative predictors of malignancy and unfavorable pathology for clinical T1a tumors treated with partial nephrectomy: a multi-institutional analysis. Urol Oncol 2015;33(112):e9-14.

[48] Kwon T, Jeong IG, Ryu J, et al. Renal function is associated with nephrometry score after partial nephrectomy: a study using diethylene triamine penta-acetic acid (DTPA) renal scanning. Ann Surg Oncol 2015;22(Suppl 3):S1594-600.

[49] Li M, Gao Y, Cheng J, et al. Diameter-axial-polar nephrometry is predictive of surgical outcomes following partial nephrectomy. Medicine (Baltimore) 2015;94:e1228.

[50] Kriegmair MC, Mandel P, Moses A, Bolenz C, Michel MS, Pfalzgraf D. Zonal NephRo score: external validation for predicting complications after open partial nephrectomy. World J Urol 2016;34:545-51.

[51] Kriegmair MC, Mandel P, Rathmann N, Diehl SJ, Pfalzgraf D, Ritter M. Open partial nephrectomy for high-risk renal masses is associated with renal pseudoaneurysms: assessment of a severe procedurerelated complication. Biomed Res Int 2015;2015:981251.

[52] Sharma P, McCormick BZ, Zargar-Shoshtari K, Sexton WJ. Is surgeon intuition equivalent to models of operative complexity in determining the surgical approach for nephron sparing surgery? Indian J Urol 2016;32:124-31.

[53] Raheem AA, Alatawi A, Kim DK, et al. Outcomes of high-complexity renal tumours with a Preoperative Aspects and Dimensions Used for an Anatomical (PADUA) score of $\geq 10$ after robot-assisted partial nephrectomy with a median 46.5-month follow-up: a tertiary centre experience. BJU Int 2016;118:770-8.

[54] Schiavina R, Novara G, Borghesi M, et al. PADUA and R.E.N.A.L. nephrometry scores correlate with perioperative outcomes of robot-assisted partial nephrectomy: analysis of the Vattikuti Global Quality Initiative in Robotic Urologic Surgery (GQI-RUS) database. BJU Int 2017;119:456-63.

[55] Ricciardulli S, Ding Q, Zhang X, et al. Evaluation of PADUA score as predictor of warm ischemia time (WIT) during laparoscopic partial nephrectomy (LPN). Urologia 2016;83:194-9.

[56] Moskowitz EJ, Paulucci DJ, Reddy BN, et al. Predictors of medical and surgical complications after robot-assisted partial nephrectomy: an analysis of 1139 patients in a multi-institutional kidney cancer database. J Endourol 2017;31:223-8.

[57] Kara Ö, Maurice MJ, Mouracade P, et al. When partial nephrectomy is unsuccessful: understanding the reasons for conversion from robotic partial to radical nephrectomy at a tertiary referral center. J Urol 2017;198:30-5.
[58] Martin L, Rouviere O, Bezza R, et al. Mayo Adhesive Probability Score is an independent computed tomography scan predictor of adherent perinephric fat in open partial nephrectomy. Urology 2017;103:124-8.

[59] Kriegmair MC, Hetjens S, Mandel P, et al. Tumor size and invasiveness matters for partial nephrectomy: External validation and modification of the Arterial Based Complexity score. J Surg Oncol 2017; $115: 768-74$.

[60] Matos AC, Dall'Oglio MF, Colombo Jr JR, Crippa A, Juveniz JAQ, Argolo FC. Predicting outcomes in partial nephrectomy: is the renal score useful? Int Braz J Urol 2017;43:422-31.

[61] Gu L, Ma X, Li H, et al. External validation of the Arterial Based Complexity $(\mathrm{ABC})$ scoring system in renal tumors treated by minimally invasive partial nephrectomy. J Surg Oncol 2017;116:507-14.

[62] Tornberg SV, Kilpeläinen TP, Järvinen P, et al. Renal tumor invasion depth and diameter are the two most accurate anatomical features regarding the choice of radical versus partial nephrectomy. Scand J Surg 2018;107:54-61.

[63] Takagi T, KondoT, Yoshida K, et al. Comparison of kidney function in the early postoperative period in transperitoneal robot-assisted laparoscopic partial nephrectomy between anterior and posterior renal tumors: a propensity score-matched study. J Endourol 2018;32:111-5.

[64] Correa AF, Toussi A, Amin M, et al. Small renal masses in close proximity to the collecting system and renal sinus are enriched for malignancy and high Fuhrman grade and should be considered for early intervention. Clin Genitourin Cancer 2018;16:e729-33.

[65] Draeger DL, Sievert KD, Hakenberg OW. Critical evaluation of the PADUA score in a retrospective analysis of open partial nephrectomy. Turk J Urol 2018;44:208-12.

[66] Petros FG, Keskin SK, Yu KJ, et al. Intraoperative conversion from partial to radical nephrectomy: incidence, predictive factors, and outcomes. Urology 2018;116:114-9.

[67] Khene ZE, Peyronnet B, Kocher NJ, et al. Predicting morbidity after robotic partial nephrectomy: the effect of tumor, environment, and patient-related factors. Urol Oncol 2018;36(338):e19-26.

[68] Wang Z, Liu C, Chen R, et al. Will the kidney function be reduced in patients with renal cell carcinoma following laparoscopic partial nephrectomy? Baseline eGFR, warm ischemia time, and RENAL nephrometry score could tell. Urol Oncol 2018;36(498):e15-24.

[69] Ficarra V, Crestani A, Bertolo R, et al. Tumour contact surface area as a predictor of postoperative complications and renal function in patients undergoing partial nephrectomy for renal tumours. BJU Int 2019;123:639-45.

[70] Yu YD, Nguyen NH, Ryu HY, Hong SK, Byun SS, Lee S. Predictors of renal function after open and robot-assisted partial nephrectomy: a propensity score-matched study. Int J Urol 2019;26:377-84.

[71] Antonelli A, Cindolo L, Sandri M, et al. Predictors of the transition from off to on clamp approach during ongoing robotic partial nephrectomy: data from the CLOCK randomized clinical trial. J Urol 2019;202:62-8

[72] Qian J, Jiang J, Li P, et al. Factors influencing the feasibility of segmental artery clamping during retroperitoneal laparoscopic partial nephrectomy. Urology 2019;129:92-7.

[73] Bertolo R, Autorino R, Simone G, et al. Outcomes of robot-assisted partial nephrectomy for clinical T2 renal tumors: a multicenter analysis (ROSULA Collaborative Group). Eur Urol 2018;74:226-32.

[74] Mir MC, Derweesh I, Porpiglia F, Zargar H, Mottrie A, Autorino R. Partial nephrectomy versus radical nephrectomy for clinical T1b and T2 renal tumors: a systematic review and meta-analysis of comparative studies. Eur Urol 2017;71:606-17.

[75] Buffi NM, Saita A, Lughezzani G, et al. Robot-assisted partial nephrectomy for complex (PADUA Score $\geq 10$ ) tumors: techniques and results from a multicenter experience at four high-volume centers. Eur Urol. In press. https://doi.org/10.1016/j.eururo.2019.03.006. 
[76] Kriegmair MC, Mandel P, Moses A, et al. Defining renal masses: comprehensive comparison of RENAL, PADUA, NePhRO, and CIndex score. Clin Genitourin Cancer 2017;15:248-55, e1.

[77] Potretzke AM, Knight BA, Zargar H, et al. Urinary fistula after robotassisted partial nephrectomy: a multicentre analysis of 1 791 patients. BJU Int 2016;117:131-7.

[78] Thiel DD, Davidiuk AJ, Meschia C, et al. Mayo Adhesive Probability Score is associated with localized renal cell carcinoma progressionfree survival. Urology 2016;89:54-60.

[79] Aparicio LM, Villaamil VM, Calvo MB, et al. Glucose transporter expression and the potential role of fructose in renal cell carcinoma: a correlation with pathological parameters. Mol Med Rep 2010;3:575-80.

[80] Husain FZ, Rosen DC, Paulucci DJ, Sfakianos JP, Abaza R, Badani KK. R.E.N.A.L. nephrometry score predicts non-neoplastic parenchymal volume removed during robotic partial nephrectomy. J Endourol 2016;30:1099-104.

[81] Lee Z, Uzzo R, Asghar A, et al. Functional parenchymal volumebased spectrum score is able to quantify ischemic injury after partial nephrectomy. Urology 2018;120:150-5. 